\title{
Tendência Temporal de Internações por Diagnóstico Oncológico em Crianças e Adolescentes
}

doi: https://doi.org/10.32635/2176-9745.RBC.2020v66n4.1010

Temporal Trend of Hospitalizations for Cancer Diagnosis in Children and Adolescents

Tendencia Temporal de las Hospitalizaciones por Diagnóstico de Cáncer en Niños y Adolescentes

Luisa Pradié Algayer'; Larissa Luma Tomasi Febras²; Bruna Segabinazzi Scheid³; Jordana Ferreira Signori; ${ }^{4}$ Leonardo Bigolin Jantsch $^{5}$

RESUMO

Introduçáo: $\mathrm{O}$ câncer pediátrico é considerado uma patologia rara, porém requer longos períodos de tratamento em centros de especialidade oncológica. Um dos principais desafios das famílias é o distanciamento dos locais de tratamento e de suporte hospitalar para internações em oncologia pediátrica. Objetivo: Analisar a tendência temporal por locais de residência e internaçâo de crianças e adolescentes por diagnósticos oncológicos, entre os anos de 1998 e 2018, no Estado do Rio Grande do Sul, Brasil. Método: Trata-se de um estudo epidemiológico documental analítico, com coleta de dados secundários em base de dados de domínio público. O estudo possui caráter de série histórica e de distribuição espaço-geográfica, das internaçôes hospitalares de crianças em tratamento oncológico no Rio Grande do Sul. Resultados: Menores de 4 anos representaram a principal demanda de internaçáo por diagnóstico oncológico infantojuvenil ao longo dos anos. Em cerca de $80 \%$ das regiôes em saúde, os pacientes acabam internando, por diagnóstico oncológico, fora das suas regiōes de saúde e locais de residência. Nos últimos 20 anos, houve aumento da prevalência nas internações, ainda muito localizadas em centros de especialidade. Conclusão: Há necessidade de intervenção de políticas públicas e descentralização de serviços especializados no tratamento e internaçáo em oncologia pediátrica.

Palavras-chave: Neoplasias/epidemiologia; Hospitalização/tendências; Fatores de Tempo; Criança; Adolescente.

\section{ABSTRACT}

Introduction: Pediatric cancer is considered a rare pathology, but it requires long periods of treatment in an oncology specialty center. One of the main challenges for the families is the distancing from treatment and hospital support facilities for hospitalizations in pediatric oncology. Objective: To analyze the time trend per places of residence and hospitalization of children and adolescents for cancer diagnoses occurred between 1998 and 2018 in the state of Rio Grande do Sul, Brazil. Method: Analytical documental epidemiological study, with collection of secondary data in a public domain database. The study is characterized as a historical series and spatial-geographical distribution of hospital admissions of children undergoing cancer treatment in Rio Grande do Sul. Results: Children under 4 years were the main demand for hospitalization for child and juvenile cancer diagnosis over the years. In about $80 \%$ of the health regions, patients end up admitted for cancer diagnosis out of their health regions and places of residence. In the last 20 years, there has been an increase in the prevalence of hospitalizations, which are still much concentrated in specialty centers. Conclusion: It is necessary the intervention of public policies and decentralization of specialized services for the treatment and hospitalization in pediatric oncology.

Key words: Neoplasms/epidemiology; Hospitalization/trends; Time Factors; Child; Adolescent.

\section{RESUMEN}

Introducción: El cáncer pediátrico se considera una patología rara, pero requiere largos períodos de tratamiento en un centro especializado en oncología. Uno de los principales desafíos para las familias es el distanciamiento de los sitios de tratamiento y apoyo hospitalario para hospitalizaciones en oncología pediátrica. Objetivo: Analizar la tendencia temporal por lugares de residencia y hospitalización de niños y adolescentes para diagnósticos de cáncer, entre los años 1998 y 2018, en el Estado de Rio Grande do Sul, Brasil. Objetivo: Analizar la tendencia temporal por lugares de residencia y hospitalización de niños y adolescentes para diagnósticos de cáncer, entre los años 1998 y 2018, en el Estado de Rio Grande do Sul, Brasil. Método: Estudio epidemiológico documental analítico, con recopilación de datos secundarios en una base de datos de dominio público. El estudio tiene el carácter de una serie histórica y de distribución espacial-geográfica, de ingresos hospitalarios de niños sometidos a tratamiento contra el cáncer en Rio Grande do Sul. Resultados: Los niños menores de 4 años representan la principal demanda de hospitalización por diagnóstico de cáncer para niños y adolescentes a lo largo de los ańos. En aproximadamente el 80\% de las regiones de salud, los pacientes terminan internando, debido al diagnóstico de cáncer, en sus regiones de salud y lugares de residencia. En los últimos 20 años, ha habido un aumento en la prevalencia de hospitalizaciones, que todavía están muy ubicadas en centros especializados, en cinco de las siete macro regiones analizadas. Conclusión: Existe la necesidad de intervención de políticas públicas y descentralización de servicios especializados en el tratamiento y hospitalización en oncología pediátrica.

Palabras clave: Neoplasias/epidemiologia; Hospitalización/tendências; Factores de Tiempo; Nińo; Adolescente.

'Universidade Federal de Santa Maria (UFSM). Palmeira das Missões (RS), Brasil. Orcid iD: https://orcid.org/0000-0003-4738-2299 ${ }^{2}$ UFSM. Palmeira das Missões (RS), Brasil. Orcid iD: https://orcid.org/0000-0003-2409-0306 ${ }^{3}$ UFSM. Palmeira das Missões (RS), Brasil. Orcid iD: https://orcid.org/0000-0002-9331-8100 ${ }^{4}$ UFSM. Palmeira das Missões (RS), Brasil. Orcid iD: https://orcid.org/0000-0002-8970-7517 ${ }^{5}$ Departamento de Ciências da Saúde da UFSM. Palmeira das Missões (RS), Brasil. Orcid iD: https://orcid.org/0000-0002-4571-183X Endereço para correspondência: Leonardo Bigolin Jantsch. Avenida Independência, 3175 - Vista Alegre. Palmeira das Missões (RS), Brasil. CEP 98300-000. E-mail: leo_jantsch@hotmail.com 


\section{INTRODUÇÃO}

Atualmente, o câncer é uma doença crônica considerada um complexo problema de saúde pública, representando a segunda causa de óbitos no Brasil. Sua fisiopatologia é provocada pela multiplicação desordenada de células, na maioria das vezes jovens, que dáo origem a tumores e substituem as células normais de um ou mais órgãos e, assim, prejudicam suas funçóes vitais ${ }^{1}$.

Os dados epidemiológicos dos casos de neoplasias no Brasil repercutem na sua alta taxa de mortalidade, visto que $21 \%$ das mortes em decorrência das doenças crônicas não transmissíveis estiveram relacionadas às neoplasias malignas. A distribuição da incidência por Regiāo geográfica brasileira reforça uma disparidade local, visto que as Regióes Sul e Sudeste concentram 70\% da ocorrência de casos novos ${ }^{1}$.

Em uma perspectiva pediátrica, segundo o Instituto Nacional de Câncer José Alencar Gomes da Silva (INCA)², o câncer infantil corresponde a um grupo de várias doenças, que têm em comum a proliferação descontrolada de células anormais e que pode ocorrer em qualquer local do organismo, em crianças e adolescentes até 19 anos. O câncer infantil é considerado uma patologia rara, relativamente, por atingir uma em cada dez mil crianças de 0 a 14. Porém, de acordo com o INCA, no Brasil, o câncer representa a primeira causa de óbitos em crianças e adolescentes. Estima-se que, no ano de 2020, 8.460 novos casos de neoplasias malignas sejam diagnosticados na população infantojuvenil ${ }^{2}$.

Estudos internacionais e nacionais destacam que as neoplasias mais comuns na infância são as leucemias, os tumores de sistema nervoso central e os linfomas ${ }^{1}$. Ao contrário do câncer em adultos, o câncer infantojuvenil normalmente afeta as células do sistema sanguíneo e os tecidos de sustentação ${ }^{2}$.

No Brasil, entre 2018-2019, ocorreram 420 mil casos novos de câncer. De acordo com os dados do Registros de Câncer de Base Populacional (RCPB) ${ }^{3}$, o percentual mediano de tumores para essa faixa etária é de 3\%. Estima-se que ocorreram 12.500 casos de câncer em crianças e adolescentes até os 19 anos. Nessas proporçôes, foram esperados, na Regiáo Sul do Brasil, 1.300 casos novos, nesse período. Para países em desenvolvimento, a proporção para o câncer infantil representa de 3\% a 10\% do total de neoplasias; em países desenvolvidos, diminui para $1 \%{ }^{3}$.

De forma inicial, um dos principais entraves no itinerário do tratamento é o diagnóstico precoce tardio ${ }^{4}$. Diferente dos cânceres em adultos, os fatores de risco relacionados com o estilo de vida não influenciam nas probabilidades de a criança e o adolescente desenvolverem câncer. Ocasionalmente, crianças podem apresentar alteraçóes genéticas que as tornem propensas a ter algum tipo de câncers.

Mesmo após diagnóstico oncológico na infância, a criança e sua família percorrem diversos cenários de atenção e passam por profissionais que irão definir o percurso terapêutico, por meio da definiçáo do protocolo adotado. Os protocolos visam à uniformização de métodos diagnósticos e tratamento do câncer da infância e adolescente e proporcionam meios de avaliação na sobrevivência, toxicidade e adesáo ao tratamento. Em sua maioria, exigem comparecimento frequente aos centros especializados, em grandes centros ou macrorregiôes de saúde, o que impóe aos pais a ausência constante no emprego e nas rotinas sociais para acompanhar a criança, gerando impacto econômico e social na família ${ }^{6}$.

Em decorrência do distanciamento dos locais de tratamento e de suporte hospitalar para internaçóes em oncologia pediátrica, durante o tratamento ou por decorrência deste, os pais, principais cuidadores, apresentam dificuldades financeiras para custear a viagem e a estadia aos grandes centros de assistência médica especializada para seu tratamento e internação, quando necessária. Essa condição reitera a necessidade de acesso aos serviços de saúde, tendo em vista a demanda associada à doença e o protocolo utilizado ${ }^{6}$.

Nessa perspectiva, fica evidente reconhecer a tendência temporal de internaçóes em decorrência de diagnósticos oncológicos em crianças e adolescentes, bem como locais de maior prevalência de local de internação e origem desses pacientes em tratamento oncológico. Discorrer sobre essa organização geográfica permite estabelecer prioridades para os centros de atenção e descentralizá-los, com intuito de minimizar o distanciamento, os custos e o impacto social do tratamento oncológico. Para tanto, o presente estudo tem por objetivo analisar a tendência temporal e os locais de origem e internação da criança e do adolescente por diagnósticos oncológicos, entre os anos de 1998 e 2018, no Estado do Rio Grande do Sul, Brasil.

\section{MÉTODO}

Trata-se de um estudo epidemiológico documental analítico, com coleta de dados secundários em base de dados de domínio público e possui caráter de série histórica e de distribuição espaço-geográfica das internaçôes hospitalares de crianças em tratamento oncológico no Rio Grande do Sul. A população do estudo foi de crianças e adolescentes da faixa etária de 0 a 19 anos e estabelecida pelo recorte realizado pelo próprio banco de dados utilizado na análise. O Departamento de Informática do Sistema Único de Saúde (DATASUS) 
utiliza, para a classificação, a faixa etária que corresponde ao período infantojuvenil de 0 a 19 anos.

Os dados foram coletados por meio do banco de dados DATASUS, sistema on-line, disponível em: http:// tabnet.datasus.gov.br/cgi/deftohtm.exe?sih/cnv/mrrs.def., na página TabNet. A seleção de busca seguiu os seguintes passos: TABNET $\rightarrow$ Epidemiológicas e morbidades $\rightarrow$ morbidade hospitalar do SUS $\rightarrow$ Geral por local de internação (1998-2018) e Geral por local de residência (1998-2018).

Foram utilizados os filtros: local de residência (Brasil e Rio Grande do Sul); capítulo CID 10: II neoplasias (tumores); e faixa etária (0-19 anos). Os dados brutos foram coletados de forma manual e transcritos para tabela do programa Excel (versão 2010). Cálculos populacionais, para análise de prevalência, foram colhidos no site do Instituto Brasileiro de Geografia e Estatística (IBGE). Os Censos 2000 e 2010 serviram de base para cálculo da prevalência.

Foi utilizada a fórmula: $\mathrm{p}=$ número de indivíduos afetados em um determinado momento/total de indivíduos, para o cálculo de prevalência.

Os dados foram analisados sob análise estatística simples (cálculo de prevalência e análise descritiva simples) e analítica, por meio da correlação da prevalência ao longo dos anos (correlação de Pearson - dados não paramétricos -, utilizando $\mathrm{p}<5 \%$ para associaçâo estatística na correlação). Quanto à distribuição espacial da diferença entre local de internação e residência, esta foi comparada após cálculos da prevalência (de tratamento e origem, índice de prevalência para cada mil habitantes da faixa etária). Os dados populacionais foram estipulados para o ano de 2018, conforme previsóes para o Rio Grande do Sul (IBGE). Os locais de residência e internação foram classificados segundo as microrregióes e macrorregióes de saúde do Estado do Rio Grande do Sul, distribuídas e classificadas conforme o Quadro 1.

Os aspectos éticos foram respeitados por meio da Resolução no ${ }^{\circ} .510$, de 7 de abril de $2016^{7}$, a qual estabelece normativas para pesquisas nas ciências humanas e sociais. Assim, por se tratar de busca em base de dados de domínio público, o presente estudo não carece de avaliação. É válido destacar o compromisso dos pesquisadores quanto à fidedignidade dos dados e à transposiçáo destes para os resultados aqui apresentados.

\section{RESULTADOS}

Os dados são apresentados sob distribuição da tendência temporal e características definidoras, conforme local de internação e residência, bem como distribuição por faixa etária. No Gráfico 1, destaca-se a tendência
Quadro 1. Descrição das microrregiões em Saúde do Estado do Rio Grande do Sul

\begin{tabular}{|c|c|c|}
\hline Macrorregião & Número & Microrregião \\
\hline \multirow{3}{*}{ Centro-Oeste } & 01 & Verdes Campos \\
\hline & 02 & Entre Rios \\
\hline & 03 & Fronteira Oeste \\
\hline \multirow{7}{*}{ Metropolitana } & 04 & Belas praias \\
\hline & 05 & Bons Ventos \\
\hline & 06 & Paranhanha \\
\hline & 07 & Vale dos Sinos \\
\hline & 08 & Vale do Caí \\
\hline & 09 & Carbonífera \\
\hline & 10 & $\begin{array}{l}\text { Porto Alegre/ } \\
\text { Metropolitana }\end{array}$ \\
\hline \multirow{4}{*}{ Missioneira } & 11 & Sete Povos das Missões \\
\hline & 12 & Portal das Missões \\
\hline & 13 & Região da Diversidade \\
\hline & 14 & Fronteira Noroeste \\
\hline \multirow{6}{*}{ Norte } & 15 & Caminho das Águas \\
\hline & 16 & Alto Uruguai Gaúcho \\
\hline & 17 & Região do Planalto \\
\hline & 18 & Região das Araucárias \\
\hline & 19 & Região do Botucaraí \\
\hline & 20 & Rota da Produção \\
\hline \multirow{2}{*}{ Sul } & 21 & Região Sul \\
\hline & 22 & Pampa \\
\hline \multirow[t]{4}{*}{ Serra } & 23 & Caxias \\
\hline & 24 & $\begin{array}{c}\text { Campos de Cima da } \\
\text { Serra }\end{array}$ \\
\hline & 25 & Vinhedos \\
\hline & 26 & Uvas e Vales \\
\hline \multirow{4}{*}{ Vales } & 27 & Jacuí/Centro \\
\hline & 28 & Santa Cruz do Sul \\
\hline & 29 & Vale das Montanhas \\
\hline & 30 & Vale da Luz \\
\hline
\end{tabular}

Fonte: Conselho das Secretarias Municipais de Saúde do Rio Grande do Sul ${ }^{8}$.

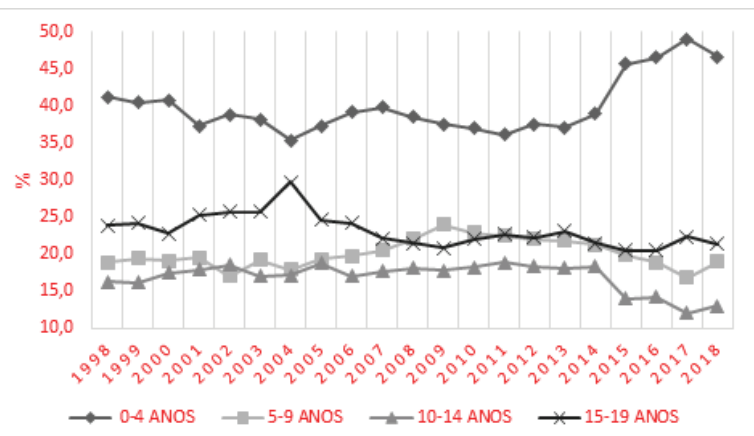

Gráfico 1. Distribuição da frequência relativa, por faixa etária, das internações hospitalares de crianças e adolescentes por diagnóstico oncológico no Estado do Rio Grande do Sul, 1998-2018 
temporal das internaçôes por diagnóstico oncológico de crianças e adolescentes no Estado do Rio Grande do Sul, estratificada por faixa etária.

Os menores de 4 anos foram os mais prevalentes nas internaçôes ao longo dos 20 anos analisados. Destaca-se que há um aumento, nos últimos cinco anos, para faixa etária menor de 4 anos. A faixa etária dos 10-14 anos apresentou queda, especialmente nos últimos três anos analisados, as demais faixas etárias permaneceram lineares, com correlaçáo fraca estabelecida $(\mathrm{IC}<0,3)$ e não significativa.

No que tange ao sexo, há uma prevalência do sexo masculino nas internaçóes, o que representa 52,9\% do total; $47,1 \%$ das internaçóes foram do sexo feminino no período 1998 a 2018. A Tabela 1 mostra a distribuiçâo por macrorregião e local de residência.

Nas macrorregiốes Norte e Missioneira, as proporçôes caíram cerca de $4 \%$ ao longo dos últimos 20 anos; para tanto, houve crescimento expressivo, para as Regióes Metropolitana e Sul, haja vista que Regiáo Sul quase dobrou o número de casos. Pela análise da distribuição da frequência de internação por macrorregião de saúde, nos últimos 20 anos, cabe destacar que houve aumento do número de internaçóes, por local de residência nas macrorregiōes 01 (IC 0,982), 02 (IC 0,826), 05 (IC 0,759), 06 (IC 0,826) e 07 (IC 0,973), porém com diferença não significativa $(\mathrm{p}<0,05)$. Já nas macrorregiōes 03 e 04 , houve diminuição da prevalência, com índices de correlação forte e dados não significativos $(\mathrm{p}<0,05)$.

$\mathrm{Na}$ Tabela 2, destaca-se a diferença entre prevalência dos locais de internação e residência de crianças e adolescentes em tratamento oncológico, distribuída por local de internação e local de residência, estratificada por faixa etária.

Compreende-se que, quanto menor a relação entre o local de internaçáo e o local de residência, maior é a necessidade das crianças da microrregiáo em se deslocarem de seu município para internar. As microrregióes 16, 18 e 20 apresentam menor relação entre o número de internados para a demanda. Cabe destacar a alta prevalência na Regiáo 16 e a necessidade de deslocamento de muitos pacientes. Todavia, as microrregióes com maior prevalência por local de internação, 01,10 e 17, são consideradas centros de saúde regionais e de referência.

Das 30 microrregiôes, $10 \%$ atendem sua demanda e acolhem nos seus serviços demandas de atendimento. Duas microrregiốes dão conta da demanda (LI-LR=0) e $83 \%$ dos municípios possuem maior prevalência de crianças em tratamento oncológico que internam para sua própria demanda de internação local. A Tabela 3 apresenta a distribuição por faixa etária.

Menores de 4 anos representam a principal demanda de internação por diagnóstico oncológico no Rio Grande do Sul, no ano de 2018, significando mais do que o dobro da média do Estado. A faixa etária de 10 a 14 anos é a menos prevalente nos serviços de internação.

\section{DISCUSSÃO}

A magnitude da carga global do câncer na infância e na adolescência permanece pouco quantificada. Não há estimativas globais de incidência, sobrevivência e mortalidade para crianças com câncer na maioria dos países de baixa renda?. O INCA destaca que o câncer infantil tem cerca de 12 mil novos diagnósticos a cada ano, com pico de incidência na faixa de 4 a 5 anos e um segundo pico entre 16 e 18 anos no Brasil. Esse dado, referente ao pico no diagnóstico, difere do percentual de internaçóes apresentado pelo estudo, à medida que a prevalência maior de internações está na faixa etária de $0 \mathrm{a}$ 4 anos ${ }^{1}$. O estudo de Silva et al. ${ }^{10}$ corrobora esses achados

Tabela 1. Distribuição do número de internações por diagnóstico oncológico em crianças e adolescentes nas macrorregiões de saúde do Estado do Rio Grande do Sul, 1998-2018

\begin{tabular}{cccccccccc}
\hline Ano & & MR01 & MR02 & MR03 & MR04 & MR05 & MR 06 & MR07 & Total \\
\hline \multirow{2}{*}{2018} & $\mathrm{n}$ & 417 & 1.337 & 3.614 & 3.309 & 1.735 & 268 & 308 & 10.988 \\
& $\%$ & 3,8 & 12,2 & 32,9 & 30,1 & 15,8 & 2,4 & 2,8 & 100 \\
\multirow{2}{*}{2008} & $\mathrm{n}$ & 416 & 1.746 & 4.401 & 4.276 & 2.322 & 341 & 317 & 13.819 \\
& $\%$ & 3,0 & 12,6 & 31,8 & 30,9 & 16,8 & 2,5 & 2,3 & 100 \\
\multirow{2}{*}{1998} & $\mathrm{n}$ & 232 & 1.294 & 6.202 & 6.540 & 1.951 & 221 & 183 & 16.623 \\
& $\%$ & 1,4 & 7,8 & 37,3 & 39,3 & 11,7 & 1,3 & 1,1 & 100 \\
\multirow{2}{*}{$\mathbf{1 9 9 8 - 2 0 1 8}$} & IC & 0,982 & 0,826 & $-0,756$ & $-0,903$ & 0,759 & 0,826 & 0,973 & \\
& $\mathrm{p}^{*}$ & 0,121 & 0,381 & 0,454 & 0,283 & 0,452 & 0,381 & 0,149 & \\
\hline
\end{tabular}

Legendas: IC: Índice de correlaçăo; MR01: Centro-Oeste; MR02: Metropolitana; MR03: Missioneira; MR04: Norte; MRO5: Sul; MRO6: Serra; MR07: Vales, *p-valor. 
Tabela 2. Diferença entre prevalência, por local de internação e residência de crianças e adolescentes em tratamento oncológico nas microrregiões de saúde do Estado do Rio Grande do Sul, em 2018

\begin{tabular}{|c|c|c|c|}
\hline \multirow[t]{2}{*}{ Microrregião } & $\begin{array}{l}\text { Local de } \\
\text { residência } \\
\text { (LR) }\end{array}$ & $\begin{array}{l}\text { Local de } \\
\text { internação } \\
\text { (ㄴII) }\end{array}$ & LI-LR \\
\hline & \multicolumn{3}{|c|}{ Prevalência/mil habitantes } \\
\hline 01 & 0,4 & 3,6 & 3,2 \\
\hline 02 & 0,9 & 0 & $-0,9$ \\
\hline 03 & 0,4 & 0,1 & $-0,3$ \\
\hline 04 & 1,1 & 0,1 & -1 \\
\hline 05 & 0,4 & 0,1 & $-0,3$ \\
\hline 06 & 0,3 & 0,1 & $-0,2$ \\
\hline 07 & 0,9 & 0,1 & $-0,8$ \\
\hline 08 & 1 & 0,2 & $-0,8$ \\
\hline 09 & 0,8 & 0,1 & $-0,7$ \\
\hline 10 & 1,1 & 2,9 & 1,8 \\
\hline 11 & 1,2 & 0,2 & -1 \\
\hline 12 & 1,1 & 0,3 & $-0,8$ \\
\hline 13 & 1,3 & 0,3 & -1 \\
\hline 14 & 49,8 & 48,6 & $-1,2$ \\
\hline 15 & 1 & 0,2 & $-0,8$ \\
\hline 16 & 42,7 & 38,3 & $-4,4$ \\
\hline 17 & 1,3 & 4,8 & 3,5 \\
\hline 18 & 1,4 & 0,1 & $-1,3$ \\
\hline 19 & 1,2 & 0,1 & $-1,1$ \\
\hline 20 & 1,4 & 0,1 & $-1,3$ \\
\hline 21 & 0,9 & 0,2 & $-0,7$ \\
\hline 22 & 0,6 & 0 & $-0,6$ \\
\hline 23 & 0,9 & 0,9 & 0 \\
\hline 24 & 0,9 & 0,2 & $-0,7$ \\
\hline 25 & 0,8 & 0,2 & $-0,6$ \\
\hline 26 & 0,4 & 0 & $-0,4$ \\
\hline 27 & 1 & 0 & -1 \\
\hline 28 & 0,8 & 0,2 & $-0,6$ \\
\hline 29 & 1,2 & 1,2 & 0 \\
\hline 30 & 1,3 & 0,1 & $-1,2$ \\
\hline
\end{tabular}

pelo maior desenvolvimento de câncer e pela necessidade de internação nas faixas etárias mais precoces ${ }^{10}$.

O câncer infantojuvenil representou 3\% de todas as neoplasias atendidas pelos hospitais de referência em tratamento oncológico, do total de internaçóes no Brasil, por tratamento oncológico, no SUS. Os diagnósticos mais frequentes foram as leucemias (30,6\%), linfomas $(16,6 \%)$ e sistema nervoso central (9,3\%). Essas internações geralmente acontecem em centros especializados, habilitados em oncologia pediátrica ${ }^{11}$.
O número de internaçôes pode retratar e caracterizar, além do perfil hospitalar, as características de crianças e adolescentes que adoecem no Estado. Diante disso, os números deste estudo salientam que a prevalência de internação por câncer infantil no Estado do Rio Grande do Sul é mais alta no sexo masculino, dado este que se assemelha a resultados realizados na China, Estados Unidos, Canadá e Brasil ${ }^{12}$.

A incidência de muitos cânceres infantis relatados desde a década de 1970 é real e particularmente notável para maior prevalência da leucemia, tipo de câncer de grande complexidade e com um tratamento que demanda muita atenção e investimento por parte do $S_{U S}{ }^{13}$, especialmente no que tange ao tempo de internação hospitalar. Embora a leucemia linfoide aguda possa ocorrer em qualquer idade, sua incidência é maior entre crianças de 2 a 5 anos, cerca de $70 \%$, diminuindo entre os adolescentes e adultos jovens; dos quais, as incidências de leucemias agudas são de $20 \%{ }^{9}$. Esse dado pode justificar a maior prevalência de internaçôes em menores de 4 anos.

Estudos destacam que as taxa de internaçóes foram maiores em crianças de 0 a 4 anos e adolescentes entre 15 a 19 anos; porém, ainda é incipiente afirmar ou descrever o maior índice de prevalência local. Subentende-se que, para a faixa etária menor de 4 anos, a vulnerabilidade clínica e as maiores taxas de leucemia sejam as possíveis justificativas para maior prevalência ${ }^{14}$. Essa variabilidade na incidência total de câncer infantil em todo o mundo é amplamente desconhecida, embora provavelmente esteja relacionada à exposição a fatores de risco como o contato com produtos químicos e cancerígenos (poluição do ar, fumo passivo, comida ou água potável), variaçóes genéticas e local de residência ${ }^{15}$.

Além disso, a variação geográfica dos achados indica que as maiores taxas estáo na microrregiāo Fronteira Noroeste (14) e isso pode ser afetado pela idade, status econômico ou classificação rural/urbana. Segundo os dados do governo do Estado do Rio Grande do Sul, essa microrregião possui uma participação maior da agropecuária e da indústria, relacionando essa ideia a possíveis interaçôes genético-ambiente de risco, como poluição do ar e uso de agrotóxicos ${ }^{16}$.

O quantitativo da média de volume de agrotóxicos é preocupante na microrregiâo 14 com o número de 755.57$874.75 \mathrm{~L} / \mathrm{km}^{2} /$ ano, quando comparada às microrregiōes com as menores taxas de diagnóstico de câncer infantil, Vale do Paranhana (06) e Uva/Vale (26), com o indicativo de $187.83-214.58 \mathrm{~L} / \mathrm{km}^{2} /$ ano. A associação entre exposição a agrotóxicos e desenvolvimento de câncer ainda gera polêmicas, principalmente porque os indivíduos estão submetidos a diversas substâncias, além de fatores genéticos, porém essas hipóteses não devem ser refutadas e sim melhor estudadas ${ }^{17}$. 
Tabela 3. Prevalência, por local de residência de crianças e adolescentes em tratamento oncológico nas microrregiões de saúde do Estado do Rio Grande do Sul, estratificada por faixa etária, em 2018

\begin{tabular}{|c|c|c|c|c|c|}
\hline \multirow{2}{*}{$\begin{array}{l}\text { Faixa etária (anos)/ } \\
\text { microrregião }\end{array}$} & 00-04 & 05-09 & $10-14$ & $15-19$ & Total \\
\hline & \multicolumn{5}{|c|}{ Prevalência/mil habitantes } \\
\hline 01 & 1,5 & 2,5 & 1,8 & 0,5 & 0,4 \\
\hline 02 & 0,1 & 1,3 & 1 & 1,2 & 0,9 \\
\hline 03 & 1,8 & 1,1 & 1,2 & 1,5 & 0,4 \\
\hline 06 & 1 & 1,6 & 1,1 & 0,6 & 0,3 \\
\hline 07 & 1,1 & 1,1 & 0,4 & 0,9 & 0,9 \\
\hline 08 & 1,9 & 0,7 & 0,7 & 0,6 & 1,0 \\
\hline 09 & 0,7 & 1 & 1 & 0,6 & 0,8 \\
\hline 10 & 1,6 & 1,1 & 0,9 & 0,7 & 1,1 \\
\hline 04 & 1,3 & 0,6 & 0,2 & 2,2 & 1,1 \\
\hline 05 & 0,3 & 0,1 & 0,2 & 1 & 0,4 \\
\hline 12 & 0,1 & 0,4 & 2,5 & 1,3 & 1,1 \\
\hline 11 & 1,7 & 0,7 & 1,4 & 1 & 1,2 \\
\hline 14 & 130,3 & 32,6 & 17,3 & 36,8 & 49,8 \\
\hline 13 & 0,1 & 1 & 0,6 & 3,1 & 1,3 \\
\hline 17 & 0,7 & 1,2 & 1 & 2,2 & 1,3 \\
\hline 18 & 2,1 & 0,1 & 1,3 & 1,9 & 1,4 \\
\hline 19 & 0,4 & 1,1 & 1,9 & 1,3 & 1,2 \\
\hline 20 & 3 & 2,2 & 0,4 & 0,7 & 1,4 \\
\hline 15 & 1,5 & 0,9 & 0,4 & 1,3 & 1 \\
\hline 16 & 100,3 & 23 & 19 & 39,6 & 42,7 \\
\hline 21 & 1,3 & 0,9 & 0,6 & 1 & 0,9 \\
\hline 22 & 1,3 & 0,1 & 0,8 & 0,2 & 0,6 \\
\hline 23 & 2 & 0,8 & 0,6 & 0,4 & 0,9 \\
\hline 24 & 2 & 0,3 & 0,3 & 1,2 & 0,9 \\
\hline 25 & 0,3 & 1,4 & 0,8 & 0,7 & 0,8 \\
\hline 26 & 0,2 & 0,2 & 0,8 & 0,4 & 0,4 \\
\hline 27 & 0,9 & 2,1 & 0,7 & 0,4 & 1 \\
\hline 28 & 1 & 0,4 & 0,8 & 1 & 0,8 \\
\hline 29 & 1,6 & 0,6 & 1,2 & 1,6 & 1,2 \\
\hline 30 & 1,5 & 1,1 & 1 & 1,7 & 1,3 \\
\hline$\Sigma$ & 8,8 & 2,7 & 2,1 & 3,6 & 4,0 \\
\hline
\end{tabular}

Os indicadores de mapeamento para uso dos serviços de atenção ao câncer pediátrico - hospitalizaçôes, quimioterapia e radioterapia - financiados pelo SUS mostraram indicativos de desigualdade no acesso, contudo, essa não deve ser confundida com a desproporçẫo dos serviços financiados pelo SUS. Destaca-se que, em mais de $80 \%$ das microrregióes, as crianças e adolescentes saem de suas regiôes de saúde para se internarem em decorrência do câncer. Esse dado é confirmado à medida que muitas Regiốes são compostas por maior prevalência de doentes que necessitam de internação, o que consequentemente remete à necessidade de buscar outros centros e serviços de saúde ${ }^{18}$.

$\mathrm{O}$ adoecimento do câncer e as rotinas de tratamento oncológico são complexos e causam sofrimento para toda a família. Quando se trata de crianças e adolescentes, 
a repercussáo pode ser agravada pelo entendimento da situação e pela necessidade, cada vez mais, da busca de centros especializados em saúde ${ }^{2}$. As repercussōes clínicas, exames invasivos, internaçôes hospitalares, distantes das suas cidades e realidades, fraqueza, inchaço e alopecia trazem desconforto emocional e social à criança, ao adolescente e à família. Cria-se a necessidade de reorganização da rotina, de acordo com a terapêutica, demandas hospitalares e de distanciamento ${ }^{19}$.

Entendem-se, como limitação do estudo, as incoerências e/ou subnotificações dos sistemas de saúde, uma vez que o estudo analisou dados secundários, cadastrados em plataforma virtual, bem como, sob o olhar de internaçáo no SUS, os que ocultam os dados dos sistemas privados de saúde. Entende-se também que possam existir diferenças entre as prevalências, visto que o estudo calculou a prevalência por meio dos dados de estimativa populacional dos Censos 2000 e 2010.

\section{CONCLUSÃO}

As internações por diagnóstico oncológico no Rio Grande do Sul são as mais prevalentes na faixa etária de 0 a 4 anos de idade e no sexo masculino. Houve aumento da prevalência nas internaçóes, ao longo dos anos em cinco, das sete macrorregiōes analisadas. As microrregiōes de saúde com maiores taxas de internação por diagnóstico oncológico pediátrico são Fronteira Noroeste (14) e Alto Uruguai Gaúcho (16). Além disso, mais de $80 \%$ das internaçóes acontecem fora das suas microrregiôes de saúde, o que ainda está atrelado à necessidade de deslocamentos de crianças e adolescentes e suas famílias durante o tratamento oncológico.

Este estudo contribuiu para evidenciar o aumento das taxas de internação em menores de 4 anos, população mais vulnerável e presente nos serviços, bem como a necessidade de deslocamento e nomadismo dos pacientes para internação por diagnóstico oncológico ao longo dos últimos 20 anos analisados. Nota-se, portanto, que há necessidade de intervenção de políticas públicas e descentralização de serviços especializados no tratamento e internação em oncologia pediátrica. Os números são crescentes e pressupóem a necessidade de deslocamento, gastos econômicos e mudanças nas rotinas de vida de quem reside distante do serviço especializado.

\section{CONTRIBUIÇÕES}

Luisa Pradié Algayer e Larissa Luma Tomasi Febras contribuíram substancialmente na concepção e planejamento do estudo; na obtenção, análise e interpretaçáo dos dados; assim como na redação. Bruna
Segabinazzi Scheid e Jordana Ferreira Signori contribuíram na obtenção, análise e interpretação dos dados; assim como na redação. Leonardo Bigolin Jantsch contribuiu substancialmente na concepção e planejamento do estudo; na análise e interpretação dos dados; assim como na redação e revisão crítica. Todos os autores aprovaram a versão final a ser publicada.

\section{DECLARAÇÃO DE CONFLITO DE INTERESSES}

Nada a declarar.

\section{FONTES DE FINANCIAMENTO}

Fundo de Incentivo à Pesquisa de recém-doutores (FIPE/ARD) da UFSM/2019. Edital 002/2019.

\section{REFERÊNCIAS}

1. Instituto Nacional de Câncer José Alencar Gomes da Silva. Estimativa 2020: incidência de câncer no Brasil [Internet]. Rio de Janeiro: INCA; 2019 [acesso 2020 jun 7]. Disponível em: https://www.inca.gov.br/sites/ ufu.sti.inca.local/files/media/document/estimativa-2020incidencia-de-cancer-no-brasil.pdf

2. Instituto Nacional de Câncer José Alencar Gomes da Silva. Tipo de cancer: câncer infanto-juvenil [Internet]. Rio de Janeiro: INCA; 2020 [acesso 2020 ago 2]. Disponível em: https:/www.inca.gov.br/tipos-de-cancer/ cancer-infantojuvenil

3. Instituto Nacional de Câncer José Alencar Gomes da Silva. Câncer no Brasil: dados dos registros de base populacional [Internet]. V. 4. Rio de Janeiro: INCA; 2010 [acesso 2020 ago 2]. Disponível em: https:// www.inca.gov.br/sites/ufu.sti.inca.local/files//media/ document//registro_de_base_populacional_completo. pdf

4. Lima BC, Silva LF, Góes FGB, et al. O itinerário terapêutico de famílias de crianças com câncer: dificuldades encontradas neste percurso. Rev Gaúcha Enferm. 2018;39:e20180004. doi: https://doi. org/10.1590/1983-1447.2018.20180004

5. Instituto Nacional de Câncer José Alencar Gomes da Silva. Tipos de câncer [Internet]. Rio de Janeiro: INCA; 2019 [acesso 2020 ago 2]. Disponível em: https://www. inca.gov.br/tipos-de-cancer

6. Grabois MF, Oliveira EXG, Carvalho MS. Assistência ao câncer entre crianças e adolescentes: mapeamento dos fluxos origem-destino no Brasil. Rev Saúde Pública. 2013;47(2):368-78. doi: https://doi.org/10.1590/ S0034-8910.2013047004305

7. Ministério da Saúde (BR), Conselho Nacional de Saúde. Resolução no 510, de 7 de abril de 2016. O Plenário do Conselho Nacional de Saúde em sua Quinquagésima 
Nona Reuniāo Extraordinária, realizada nos dias 06 e 07 de abril de 2016, no uso de suas competências regimentais e atribuiçóes conferidas pela Lei n o 8.080, de 19 de setembro de 1990, pela Lei n o 8.142, de 28 de dezembro de 1990, pelo Decreto n o 5.839, de 11 de julho de 2006, e [...] [Internet]. Diário Oficial da União, Brasília, DF; 2016 maio 24. Seção I, p. 44 [acesso 2020 abr 5]. Disponível em: https://www. in.gov.br/materia/-/asset_publisher/Kujrw0TZC2Mb/ content/id/22917581

8. Conselhos das Secretarias Municipais de Saúde do Rio Grande do Sul (BR) [Internet]. Porto Alegre, RS: COMSRS; 2018-2020. Regiôes de Saúde; [data desconhecida] [acesso 2020 abr 5]. Disponível em: https://www.cosemsrs.org.br/regioes-de-saude

9. Bhakta N, Force LM, Allemani C, et al. Childhood cancer burden: a review of global estimates. Lancet Oncol. 2019;20(1):e42-e53. doi: https://doi.org/10.1016/ s1470-2045(18)30761-7

10. Silva MGP, Bedor CNG, Alencar KMSA, et al. Tendências da morbimortalidade por câncer infantojuvenil em um polo de fruticultura irrigada. Cad Saúde Colet. 2018;26(1):38-44. doi: https://doi.org/10.1590/1414$462 \times 201800010477$

11. Santos MO. Incidência, mortalidade e morbidade hospitalar por câncer em crianças, adolescentes e adultos jovens no Brasil: informaçóes dos registros de câncer e do sistema de mortalidade. Rev Bras Cancerol. 2018;64(3):439-40. doi: https://doi.org/10.32635/21769745.RBC.2018v64n3.56

12. Mutti CF, Cruz VG, Santos LF, et al. Perfil clínicoepidemiológico de crianças e adolescentes com câncer em um serviço de oncologia. Rev Bras Cancerol. 2018;64(3):293-300. doi: 10.32635/2176-9745. RBC.2018v64n3.26.

13. Rodrigues JVC, Sanches AM, Oliveira ATD, et al. Leucemia e gastos hospitalares: uma análise do impacto econômico para o sistema público de saúde de Montes Claros, MG. Rev Aten Saúde. 2019;17(59):33-8. doi: https://doi.org/10.13037/ras.vol17n59.5753

14. Steliarova-Foucher E, Colombet M, Ries LAG, et al. International incidence of childhood cancer, 2001-10: a population-based registry study. Lancet Oncol. 2017;18(6):719-31. doi: https://doi.org/10.1016/ S1470-2045(17)30186-9

15. Siegel DA, Li J, Henley SJ, et al. Geographic variation in pediatric cancer incidence - United States, 2003-2014. MMWR Morb Mortal Wkly Rep. 2018;67:707-13. doi: https://doi.org/10.15585/mmwr.mm6725a2

16. SIT: sistema de informaçóes territoriais [Internet]. Brasília, DF: Secretaria de Desenvolvimento Territorial do Ministério do Desenvolvimento Agrário; [2012]. Perfil territorial: fronteira noroeste - RS; 2015 maio [acesso 2019 abr 20]. Disponível em: http://sit.mda. gov.br/download/caderno/caderno_territorial_228_ Fronteira\%20Noroeste\%20-\%20RS.pdf.

17. Jobim PFC, Nunes LN, Giugliani R, et al. Existe uma associação entre mortalidade por câncer e uso de agrotóxicos?: uma contribuição ao debate. Ciênc Saúde Coletiva. 2010;15(1):277-88. doi: https://doi. org/10.1590/S1413-81232010000100033

18. Grabois MF, Oliveira EXG, Carvalho MS. Childhood cancer and pediatric oncologic care in Brazil: access and equity. Cad Saúde Pública. 2011;27(9):1711-20. doi: https://doi.org/10.1590/S0102-311X2011000900005

19. Bicalho C, Araújo A, Botti N. Processo de adolescer relacionado ao adoecimento e tratamento do câncer. Psic Saúde Doenças. 2019;20(1):74-87. doi: https://doi. org/10.15309/19psd200106 